\title{
L'invention d'un droit colonial. Réflexion sur la pluralité des normes et sur les références au droit dans l’administration française en Algérie, 1830-1834.
}

Citation : «L'invention d'un droit colonial. Réflexion sur la pluralité des normes et sur les références au droit dans l'administration française en Algérie, 1930-1834 », in C. M. Herrera, A. Geslin, M.-C. Ponthoreau, Postcolonialisme et droit : perspectives épistémologiques, Paris, éd. Kimé, novembre 2020, p. 19-41

François Dumasy

IEP d'Aix en Provence

CHERPA/TELEMMe

L'histoire du droit en situation coloniale a connu depuis le début des années 2000 un renouvellement important, qui a bénéficié de croisements disciplinaires et offerts de nouvelles perspectives de recherche. Dans le cas de l'Algérie coloniale, elle a en grande partie relevé du travail de juristes soucieux de retracer la genèse d'un ensemble disparate de mesures et normes diverses et de faire une histoire du droit en tant qu'institution. Ce champ de recherche s'est enrichi ces dernières années d'analyses de cas localisés et d'ouvrages de synthèse visant à retrouver les logiques générales des divers droits coloniaux. Un tel apport invite d'autant plus à opérer la convergence avec les travaux des historiens et des sociologues de la colonisation, qui se sont intéressés aux pratiques et aux théories de la domination coloniale en débordant le cadre des institutions juridiques proprement dites.

Ces avancées ont nourri une réflexion sur la nature de ce droit qui a visé à interroger le hiatus entre les pratiques de la domination coloniale, marquées par l'importance de l'arbitraire, et une rhétorique juridique prétendant à la cohérence et la régularité. Loin de n'être qu'un habillage masquant mal la violence de la colonisation, le droit a pu être abordé ainsi comme un élément central de discours producteurs d'effets sociaux et économiques (notamment la discrimination entre colonisés et colons) et comme un espace de conflits dans lequel se reformulait le rapport colonial. L'intérêt renouvelé pour les approches juridiques de la situation coloniale a ainsi contribué à repenser les fondements épistémologiques d'une histoire de la colonisation désormais de plus en plus appréhendée dans une perspective de décloisonnement chronologique et spatial ${ }^{1}$.

Un des apports du champ foisonnant des études postcoloniales à cet égard a été de souligner les effets sur la longue durée des représentations et pratiques de la colonisation. Pour autant, la centralité accordée au fait colonial a aussi conduit à critiquer une vision déformante, qui a eu pour conséquence de minorer ou d'occulter « les dynamiques passées des sociétés du Maghreb » et la pluralité des temporalités ${ }^{2}$. On peut dès lors interroger les limites d'une histoire par le droit qui, intrinsèquement liée au cadre institutionnel imposé par le pouvoir colonial, comporte un risque d'écrasement de perspective. À cela s'ajoute le fait que la mise en ordre tardive du droit colonial dans le dernier quart du XIXe siècle comme discipline universitaire a contribué à populariser l'idée que les premières années de la colonisation en Algérie seraient négligeables pour la compréhension du droit colonial et, au-delà, pour celle d'une domination dont les mécanismes et la logique n'apparaitraient clairement que plus tard. La grande majorité des travaux des juristes historiens du droit s'est ainsi intéressée à une période restreinte, allant

${ }^{1}$ Cf. Blais, Fredj \& Thénault 2016 et Bertrand 2008.

${ }^{2}$ Grangaud \& Oualdi, $2016: 135$. 
de la fin du XIXe siècle à la décolonisation. Le cas est encore plus marqué pour les années 1830-1834, souvent perçues dans l'histoire de la colonisation de l'Algérie sous le seul angle des hésitations ou des incertitudes ${ }^{3}$. L'ordonnance du 10 août 1834 pose en effet les bases d'un droit colonial fondé sur la séparation des législations entre musulmans et juifs d'une part, soumis aux juridictions confessionnelles pour le statut personnel, et les Européens d'autre part, avant que les ordonnances de 1841 et 1842 ne viennent définitivement abolir la justice de type consulaire par la transposition des codes métropolitains et amorcer la limitation des compétences de la justice indigène ${ }^{4}$. Cette justice serait cependant restée grevée par la confusion des législations, l'armée se réservant un ample pouvoir disciplinaire, et par la lenteur et l'incomplétude des codifications, d'autant plus que la correspondance des commandants en chef de l'armée d'Afrique «dévoile [...] un rythme chronologique étranger à la cadence des réformes judiciaires $\iota^{5}$. Comment dès lors dépasser le constat répété de la non-application et de la confusion des législations? Quelle place faire à récurrence des notions de justice, de droit ou d'équité qui imprègnent au même moment nombre de rapports et écrits d'administrateurs, d'autant que peu d'entre eux ont une réelle culture juridique?

Appréhender la justice dans sa polysémie, comme institution, comme répertoire de valeurs et comme moyen de légitimation permet de reconsidérer la place du recours au droit précisément à un moment où le jeu des institutions judiciaires est peu défini et est concurrencé par d'autres formes d'autorité. Notre hypothèse est que ces premières années de présence française en Algérie s'inscrivent dans des interrogations plus larges sur la nature du droit dans la constitution de l'ordre politique. Elles offrent ainsi un terrain d'observation la façon dont les normes et pratiques du droit sont élaborés ou mobilisés par différents acteurs, souvent sans réelle formation juridique, dans un contexte de concurrence de compétences et d'indéfinition du pouvoir. Replacer le droit dans l'économie générale de la domination suppose par ailleurs d'en analyser les limites par rapports à d'autres formes de régulation proposées, qu'il s'agisse de la force ou du marché. Il s'agit ainsi ici de contribuer à l'histoire de la gouvernementalité coloniale en réinterrogeant les mécanismes de décision et de légitimation au plus près des acteurs locaux de l'administration. Nous reviendrons pour cela sur les termes des débats récents sur le droit colonial, avant d'analyser les différents registres auxquels puisent ces acteurs pour penser le droit son espace d'application.

\section{Postcolonial et droit : de quoi s'agit-il ?}

Si l'histoire du droit désigne à la fois une discipline institutionnalisée et un champ de recherche sur un objet, à quoi renvoie l'expression de «postcolonial »? L'idée d'une école postcoloniale en tant que telle a fait l'objet de vifs débats quant à son caractère réellement novateur, J.-F. Bayart critiquant l'illusion de la rupture et rappelant que la déconstruction du discours colonial était déjà pratiquée par des historiens contemporains des luttes d'indépendance. Le postcolonial ne serait dès lors qu'un jeu effet d'affichage visant à la captation des ressources universitaires et éditoriales ${ }^{6}$. La réponse de J. Pouchepadass, un des principaux introducteurs des études postcoloniales en France, n'en est pas moins intéressante pour ce qu'elle dit des enjeux attribués à cette approche : le postcolonial aurait permis de davantage mettre l'accent sur les systèmes de représentations comme objet de recherche en soi,

\footnotetext{
${ }^{3}$ Bontems 2014

${ }^{4}$ F. Lekéal 2005 : 16. Sur l'internement, cf. Thénault 2011.

${ }^{5}$ Ibid.

${ }^{6}$ J.-F Bayart 2010.
} 
désignant la colonisation comme un phénomène débordant le seul cadre des acteurs et imprégnant les mentalités sur la longue durée. Il aurait ainsi une utilité pédagogique, celle de démonter les impensés coloniaux toujours présents dans certains segments de l'opinion publique $^{7}$. Derrière la question de l'antériorité, ces positions illustrent l'intérêt porté aux discours et représentations dans le sillage des travaux d'E. Said depuis les années 1970. Ceuxci ne doivent dès lors plus être appréhendés comme de simples illustrations d'enjeux situés ailleurs, mais comme des éléments centraux du processus d'expansion et de domination.

Une telle approche n'est sans doute pas étrangère au renouveau de l'intérêt historiographique pour le droit. S'il a fallu, comme le note X. Rousseaux ${ }^{8}$, deux générations pour aborder de nouveaux ce domaine, ce fut aussi au prix d'un changement de perspective : celui de considérer le droit comme objet légitime de la recherche non seulement pour ses héritages actuels mais aussi comme vecteur privilégié de l'expression de la pensée coloniale. Les travaux sur le statut des métis, sur le recours au notions de « prestige » et de « dignité » ou sur le rôle du « code de l'indigénat » dans les pratiques de gouvernance colonisation ont permis de souligner l'importance des catégories juridiques et judiciaires dans l'exercice quotidien de la domination ${ }^{9}$. Dans le même temps, la justice coloniale a fait l'objet des avancées d'historiens du droit visant à retracer l'évolution générale des textes et des pratiques juridiques ${ }^{10}$, et, audelà, à « de s'intéresser à la fois au fond de la règle et à celui ou ceux qui en sont à l'origine, en tant qu'institution et en tant que personnes », autrement dit, à " ne pas se contenter uniquement $\mathrm{du}$ contenu, mais ne pas mener, pour autant, qu'un travail prosopographique $»^{11}$. Il est ainsi possible de distinguer plusieurs domaines qui, pour ce qui concerne la colonisation française, ont connu d'importants renouvellement ces dernières années. Outre la question des compétences judiciaires, que ce soit pour distinguer celles dévolues respectivement aux pouvoirs civils et militaires ou pour évaluer la capacité d'énonciation du droit au niveau individuel ou local, celle du registre juridique appliqué (droit français ou droits locaux) et celle du statut des individus, ont ainsi bénéficié d'études qui ont permis de les repenser aux niveaux métropolitains et impériaux ${ }^{12}$.

Or ces travaux ont, plus ou moins directement, contribué à interroger la notion même de droit et à questionner les sources d'une histoire de la colonisation par le droit. L'inventaire des textes de nature législative (décrets, arrêtés, ordonnances, lois, règlements etc.) a permis ainsi de mieux comprendre la diversité des législations, des acteurs et des compétences, soulignant la confusion entre certains registres disciplinaires ${ }^{13}$. Mais il a aussi montré les limites d'une approche uniquement institutionnelle du fait même des contradictions de ce corpus, jusqu'à poser la question de la représentativité du cas algérien ${ }^{14}$. Comme le montre $\mathrm{S}$. Thénault à partir de l'étude de l'internement en Algérie, «cette incertitude documentaire interdit de fonder l'histoire de l'internement sur les textes juridiques, qui ne constituent qu'un fondement mouvant» et ne peuvent être qu'un «point d'entrée dans l'histoire de la colonie et plus particulièrement dans celle de l'État », d'autant que les juristes de la période coloniale eux-

\footnotetext{
${ }^{7}$ J. Pouchepadass 2010.

${ }^{8}$ Rousseaux, op. cit.

${ }^{9}$ Saada 2002 et 2007 , Merle 2004.

${ }^{10}$ Citons parmi les travaux récents Bontems, 2014 et 2018, Bras 2015 ; Renucci 2011 ; ; Renucci \& Gérard-Loiseau 2011 ; ainsi que les travaux de l'UMR «Dynamique du droit» de l'Université de Montpellier et la collection dirigée par Bernard Durand et Martine Fabre. Cette liste n'est bien sûr par exhaustive.

${ }^{11}$ Renucci $2011: 3$.

${ }^{12}$ Cf. entre autres Blévis 2003, Cherchari 2004 ; Weil 2005 ; Saada 2006 ; Renucci 2010.

${ }^{13}$ Durand et alli 2005. La question de cette représentativité est soulevée aussi par la critique faite par P. VidalNaquet et G. Meynier aux travaux d'O. Le Cour Grandmaison en 2005. De même, la superposition de législations concernant les colonisés dans le cas de l'AOF impose de dépasser la lettre des textes pour aborder la réalité des pratiques : cf. Brunet-La Ruche \& Manière 2014.

${ }^{14}$ Durand 2005 : 46.
} 
mêmes ne parviennent pas à en élaborer des compilations exhaustives ${ }^{15}$. La constitution du droit colonial comme savoir académique illustre du reste la difficulté éprouvés par les juristes de la fin du XIXe siècle à en retrouver la cohérence ${ }^{16}$. Or, comme le montre E. Saada, le droit occupe une place centrale dans la «science coloniale» dispensée au seuil du XXe siècle aux administrateurs ${ }^{17}$.

Cette ambivalence a donné lieu à des prises de position contrastées sur la place même du droit dans la construction du rapport colonial. Un des exemples les plus connus est la critique adressée implicitement par G. Mann à l'analyse d'E. Saada sur la colonisation française en Indochine comme un «empire de la loi ». Pour celui-ci, le cas indochinois ne peut être représentatif d'une domination fondée au contraire en AOF en grande partie sur du "nondroit» (non law), si bien qu'on ne peut accepter de présenter «le régime colonial français comme étant fondamentalement un régime légal, dans lequel la loi et la procédure légale étaient centrales $\gg{ }^{18}$. Il considère dès lors la législation en AOF envers les colonisés comme une « couverture légale », un « alibi » qui n'est « ni le responsable, ni l'agent de la règle coloniale (colonial rule) », dont l'utilité n'est pas à trouver dans la colonie elle-même (qui serait soumise au contraire à l'arbitraire des agents administratifs), mais dans la nécessité de préserver l'administration locale des critiques extérieures, notamment de métropole ${ }^{19}$.

Cette position est représentative de la difficulté à définir le droit colonial. Mann n'occulte nullement l'existence d'un appareil et d'un apparat juridiques en AOF. La distinction qu'il opère entre les termes de law et de rule repose sur une différenciation procédurale mais non légale. On peut toutefois aussi à l'inverse avancer que l'arbitraire des agents de la colonisation, notamment les commandants de cercle, n'est pas hors du droit dans la mesure où c'est la législation coloniale elle-même qui les autorise à l'exercer. L'idée de " non-droit» avancée par Mann renvoie ici à une acceptation particulière du droit, reposant implicitement sur la confusion entre le droit et la justice comme institutions et comme valeurs. Encore cette vision procède-t-elle parfois par anachronisme, en reposant sur les principes de régularité et à d'intangibilité des normes (la loi est écrite, donc connaissable ; elle est la même pour tous au même moment) qui n'étaient parfois pas mêmes appliqués dans les métropoles. P. Vidal Naquet et G. Meynier ont ainsi rappelé l'analogie et la contemporanéité entre les violences commises en Europe et celles dans les colonies, appelant de fait à une approche globale des systèmes de domination sans pour autant négliger les caractères spécifiques de la colonisation ${ }^{20}$. L'étude comparée des législations montre de même la similarité des problèmes posés aux juristes en Europe et dans les empires coloniaux ${ }^{21}$.

Un des problèmes soulevés par ces débats est ainsi l'assimilation entre les notions d'exception et de normalité d'une part et entre légalité et illégalité d'autre part. Or cette exception est « revendiquée comme telle » et est pensée comme « légale et juridique » comme le montrent les débats à la Chambre lors des renouvellement du code de l'indigénat ${ }^{22}$. Elle reste aussi adossée à un discours constamment réitéré, que l'on trouve dès les débuts de la

\footnotetext{
15 Thénault $2011: 124$ et 127.

${ }^{16}$ Il faut ainsi attendre 1896 pour qu'une première systématisation soit achevée Vatin $2015: 38$ sq. Cf. aussi Saada 2009.

${ }^{17}$ Saada et 2009 .

${ }^{18}$ Mann 2009 : 333 et 338. À noter que Merle 2004 n'emploie, dans une logique proche, le terme de « légalisation » qu'entre guillemets.

${ }^{19}$ Ibid. : 338 : « Law itself was neither a culprit nor an agent of colonial rule. It was once a tool, the utility of which is evident, and an alibi ». cf. aussi p. 334.

${ }^{20}$ Meynier \& Vidal-Naquet 2015.

${ }^{21}$ Rousseaux, op. cit., note que « les chantiers du droit et de la justice aux colonies rencontrent les mêmes débats, avancées et critiques historiographiques que l'histoire du droit et de la justice dans la longue durée occidentale », se référant notamment aux luttes pour supprimer des pratiques et coutumes judiciaires alternatives en Europe occidentale au XIXe siècle, notamment en milieu rural.

${ }^{22}$ Blévis 2013 : 94-95.
} 
colonisation en Algérie : celui d'une homogénéisation à venir dès lors que les colonisés auront atteint le degré de civilisation des colonisateurs ${ }^{23}$. Que les administrateurs y aient eux-mêmes cru importe ici moins que sa fonction de fiction légitimatrice, puisque cette homogénéisation était constamment renvoyée à un avenir inatteignable à hauteur d'une vie. Il est donc nécessaire d'appréhender l'espace du droit colonial comme étant à la fois inégalitaire, inique, en partie arbitraire et enchâssé dans un dispositif légalisé, conjuguant des régimes juridiques et disciplinaires différents.

L'importance de saisir le droit dans son application a conduit à s'intéresser plus largement à la capacité des acteurs locaux à se saisir des normes et à en faire valoir leur interprétation ${ }^{24}$. L'étude du statut personnel a ainsi contribué à souligner le rôle essentiel de la jurisprudence dans un contexte juridique lacunaire ${ }^{25}$. Comme le note J. Surkis, le droit colonial est de fait en grande partie élaboré par les conflits, non en dépit d'eux. Ces contentieux « à la marge du système juridique impérial », qui se jouent aux échelons des appareils administratifs et judiciaires locaux, montrent comment s'élabore une formulation juridique d'une domination contrainte de se reformuler au fur et à mesure que des cas imprévus se présentent ${ }^{26}$. La législation coloniale n'est ainsi jamais totalement stable, d'autant qu'elle ne cesse d'être soumise aux critiques et aux débats, que ce soit au parlement ou de la part les juristes euxmêmes.

Ces apports élargissent la possibilité d'une histoire du droit qui articule les approches sociale, politique et culturelle et qui prenne en compte aussi bien l'action des administrés que des administrateurs. Elles permettent aussi de repenser l'articulation entre le local et l'impérial dans la mesure où le droit peut apparaitre comme une des voies d'expression d'un métalangage politique charriant des représentations communes. Le droit colonial peut ainsi être appréhendé aussi comme un espace de concurrence éthique, ou, pour reprendre l'expression de R. Bertrand, comme un lieu de conflits au sein d'une «polyphonie morale $»^{27}$. La prise en compte des ensembles normatifs, qu'ils soient directement liés à l'État ou hors de son contrôle direct, de même que l'insertion du droit dans une histoire des représentations permet ainsi d'ouvrir à la voie à une histoire des sensibilités ${ }^{28}$. J. Surkis rattache ainsi le droit colonial à la pensée juridique classique dont elle retient deux conceptions fondamentales sur le terrain impérial : d'une part la distinction entre droit de la personne et droit des objets, qui différencie ce qui relève du marché et ce qui relève du statut personnel, et d'autre part l'idée que la loi doit concourir à la morale sexuelle et familiale. Alors qu'en Algérie le statut personnel établit une frontière entre colonisés et colons au nom de la différence religieuse et culturelle, la propriété devient rapidement l'objet de multiples dispositifs pour la faire passer sous le droit français ${ }^{29}$. Dans ce schéma, le social - le droit civil - ne relève pas du domaine juridique commun et reste dévolu aux juridictions préexistantes, c'est-à-dire aux tribunaux musulmans et «mosaïques », prétendument coutumiers mais transformées et contrôlés en Algérie par l'autorité politique qui en restreint les compétences au cadre familial.

\footnotetext{
${ }^{23}$ Urban 2014 constate que ce discours est présent dès l'expédition d'Égypte.

${ }^{24}$ Il semble que deux voies ont été privilégiées pour cela: d'une part l'étude des carrières et pratiques des magistrats et juristes, d'autre part l'analyse de la jurisprudence et des rapports d'ordre judiciaire. On peut se rapporter pour la première à Rousseaux, op. cit., notes 36 et 37, ainsi qu'à Thénault 2001 ou Renucci 2016. Voir aussi Gobe 2013 pour une analyse cette fois sociologique des avocats en Tunisie (centrée davantage sur la période post-coloniale).

${ }^{25}$ Il revient ainsi en 1862 à la cour d'appel d'Alger d'inventer à proprement parler la différence entre nationalité et citoyenneté françaises, à rebours de la logique métropolitaine. Cf. Blévis 2003, Cherchari 2004 : 749-758; Saada $2006: 185$.

${ }^{26}$ Surkis 2017 : 144. Voir aussi, pour un plus ample développement, Surkis 2010 et 2019.

${ }^{27}$ Bertrand 2010 : 140.

${ }^{28}$ Surkis 2017 parle ainsi de « effective and affective law »

${ }^{29}$ Surkis 2017 : 238-239 et Id., 2019. Cette réflexion emprunte à celle de Dunkan Kennedy 2006.
} 


\section{La pluralité des références juridiques}

Concevoir le droit comme un champ fluctuant de concurrences plus que comme un ensemble figé invite à retracer la généalogie des représentations et des habitus juridiques des acteurs. Les approches juridiques sur les premiers temps de la conquête de l'Algérie se sont souvent limitées à rappeler les dispositions énoncées par l'autorité colonisatrice. Si une telle posture peut être pertinente pour le dernier quart du XIXe siècle, c'est-à-dire pour la période où s'affirme une idéologie coloniale structurée et diffuse, peut-elle l'être à un moment où non seulement le terme de colonisation renvoie à des pratiques différentes et fait l'objet de critiques importantes, mais où la présence française est sujette à controverses sur sa nature et ses objectifs $?^{30}$ Le risque est alors de prêter à la colonisation d'emblée un caractère exceptionnel du point de vue juridique et politique, quand bien même des pratiques similaires se retrouvent dans les cas de guerre et d'occupation sur d'autres terrains. Les ordonnances du 22 juillet 1834 et du 10 août 1834 ont ainsi été fréquemment considérées, à la suite de C. Collot, comme « les premiers jalons de l'organisation définitive française sur la base de l'assimilation en respectant la personnalité des juridictions » (l'assimilation ne concernant ici que les Européens tandis que le « respect» s'avéra rapidement discriminant) ${ }^{31}$. Jusqu'à lors, la politique en la matière avait été définie par le commandement militaire de l'Armée d'Afrique, via l'arrêté du 22 octobre 1830 considérant tout acte commis contre des Français, militaire ou non, comme relevant du conseil de guerre et plaçant la justice civile des musulmans et des juifs sous la compétence de tribunaux confessionnels, tandis que la justice consulaire était étendue aux Français et Européens en première instance pour le civil et le pénal ${ }^{32}$. L'ordonnance du 10 août 1834 confirme donc la séparation des juridictions en fonction de l'appartenance religieuse et culturelle et confirme le contrôle supérieur de l'autorité militaire ${ }^{33}$.

Or cet échafaudage juridique puise à des répertoires variés. Il se rattache, pour les compétences dévolues au commandant en chef (puis au gouverneur à partir de 1834), aux réformes introduites dans les anciennes colonies entre 1827 et $1830^{34}$. Le modèle des colonies d'Ancien régime est par ailleurs revendiqué, pour ce qui concerne la compétence des juges, par

\footnotetext{
${ }^{30}$ Pitts 2008 ; Sessions 2011.

${ }^{31}$ Collot 1987 : 170. L'ordonnance du 22 juillet place l'ancienne régence d'Alger sous souveraineté et précise l'organisation administrative du territoire. Celle du 10 août 1834 établit le service judiciaire de l'Algérie avec la mise en place à Alger, Bône et Oran de tribunaux de première instance pour les Français et Européens, ainsi que d'un tribunal de commerce et d'un tribunal supérieur, tandis qu'étaient confirmés les tribunaux musulmans et juifs pour les habitants de l'ancienne régence. Sur ces aspects, voir aussi Durand 2005 et Lekéal 2005. Sur Collot et son influence sur l'histoire du doit de l'Algérie coloniale, cf. Thénault $2011: 119$.

${ }^{32}$ Durand 2005 : 49 ; Bontems 2014 : 33. Selon l'arrêté, « la cour de Justice est autorisée à appliquer les lois françaises ou celles du royaume d'Alger, de même que les usages et coutumes de l'un ou l'autre pays, suivant ce qu'elle le croira convenable » et est compétente pour juger en appel les décisions des qadis et rabbins.

${ }^{33}$ Ibid. : le 9 juin 1831, un arrêté confie la compétence de l'appel en correctionnel au conseil d'administration présidée par le général en chef, « donnant ainsi pouvoir à l'autorité militaire de réformer les décisions de l'autorité judiciaire $\gg$.

${ }^{34}$ Durand 2005 : 70. Ces réformes sont appliquées à la Réunion (1827), aux Antilles et en Guyane (1828) et à Saint-Pierre et Miquelon (1830).
} 
l'intendant civil Louis-André Pichon en $1832^{35}$. L'arrêté du 22 octobre 1830 renvoie quant à lui à l'édit de 1778 sur la justice des Échelles du Levant ${ }^{36}$.

S'il y a bien une filiation avec les anciennes colonies, celle-ci n'est toutefois que partielle en raison de l'indétermination du statut des territoires d'Algérie, qui conduit le commandement militaire à maintenir les juridictions antérieures. L'intendant civil à Alger peut ainsi répondre le 9 octobre 1830 à la demande d'un ancien défenseur agréé au tribunal civil et de commerce de Saint-Louis du Sénégal que « le royaume d'Alger ne fait point partie de la France et n'en est pas une colonie. Jusqu'ici, il doit être régi par les mêmes règles et suivant les mêmes formes qu'auparavant. Il est donc inutile d'invoquer le Code de procédure suivi en France et même le droit commun $»^{37}$. L'Algérie n'est de fait pas comprise dans la réforme de la justice dans les colonies issues de l'Ancien Régime du 24 avril 1833, à l'instar des territoires français de l'Inde ${ }^{38}$. Officiellement, l'expédition d'Alger s'inscrit le cadre de la lutte contre la piraterie et l'esclavage instituée lors du congrès d'Aix-la-Chapelle en 1818, comme le rappelle encore le sous-intendant civil à Bône en septembre $1833^{39}$. C'est le seul objectif de mettre fin au « règne des Turcs, oppresseurs du pays depuis trois siècles » qui est par ainsi invoqué pour justifier de l'expulsion des fonctionnaires ottomans ${ }^{40}$. Hors de ce cadre, aucune instruction n'a été donnée sur l'administration et la justice des territoires occupés par l'armée, d'autant que le modèle napoléonien d'exportation du code civil n'entre pas dans l'esprit de la Restauration.

La référence à la colonisation n'est donc ni évidente ni exclusive d'autres logiques. Elle est mobilisée par le général Clauzel qui arrive à Alger le 2 septembre 1830 avec ordre, selon son témoignage, de " prendre quelques mesures pour conserver, à tout événement, des points militaires sur les côtes d'Afrique » et d'annoncer à l'armée d'Afrique 1 changement de régime en France. Selon son témoignage publié en 1831, il aurait eu en effet rapidement la conviction qu' " Alger deviendrait une importante colonie qui nous dédommagerait amplement de la perte de Saint-Domingue, et peut-être aussi des frais immenses que nous coûte l'onéreuse possession de nos autres colonies ». L'instruction du 30 octobre 1830, qu'il considère comme la preuve d'une volonté de colonisation, se borne cependant à lui demander un « examen attentif de tous les moyens d'améliorer l'état du pays, dans l'intérêt de la France combiné avec celui des indigènes $»^{41}$. L'idée de colonisation apparait aussi sous la plume de l'intendant civil Volland en janvier 1831, qui considère que l'occupation prend désormais l'apparence d'un «prélude d'une colonisation $»^{42}$.

L'incertitude des compétences du pouvoir militaire et du statut de ces territoires apparait encore nettement en août 1832, dans un jugement du conseil d'État à propos d'un litige

\footnotetext{
${ }^{35}$ Louis-André Pichon qui institue ainsi à Oran et Bône « une justice de la nature de celle qui existait autrefois dans nos colonies, dans les sénéchaussées. J'ai nommé un juge royal et un procureur du roi qui doivent, pour le civil, siéger ensemble avec un greffier, le juge prononçant le jugement. J'ai fait pour Bône une pareille organisation, par un arrêté du 20 avril 1832 [...]. On voit, dans cet arrêté que, pour le criminel, le juge et le procureur du roi, chacun en droit soi, commencent l'instruction, et envoient cette instruction et les prévenus à Alger. Cette simplicité nous est imposée par les circonstances » (Pichon 1833 : 243).

${ }^{36} \mathrm{La}$ fin des juridictions consulaires pour les ressortissants européens est demandée par le ministère des Affaires étrangères dès le 30 août. Cf. Louis-André Pichon, Alger sous la domination française, Paris, Barrois et Duprat, 1833 , p. $177-178$ et $222-223$.

${ }^{37}$ Estoublon 1890 : année 1830, p. 1-2.

${ }^{38}$ Saada $2006: 168$.

39 Rapport sur la colonisation, 21/09/1833, Archives nationales d'Outre-mer d'Aix-en-Provence (désormais ANOM), 18 MIOM 25, liasse 66.1.

${ }^{40}$ Clauzel $1831: 8$.

${ }^{41}$ Ibid., p. 8-9.

${ }^{42}$ Service historique de la Défense, 1H6, Notice sur le mode de gouvernement provisoirement établi dans le royaume d'Alger. 12/01/1831. Le texte est par la suite publié en vue de défendre publiquement la politique de Clauzel (Volland 1831). L'idée d'une occupation d'abord justifiée par la piraterie se trouve encore sous la plume du sous-intendant civil de Bône en septembre 1833.
} 
concernant un contrat signé par le général Clauzel en novembre 1830 pour la construction d'un théâtre à Alger. La demande d'indemnisation d'un entrepreneur pour non-respect du contrat pose en effet la question du droit auquel il ressort. Clauzel justifie sa dérogation au droit métropolitain par les «pouvoirs illimités, conférés [à lui] verbalement par le roi et les ministres ». Or le ministre de la Guerre considère dans le même temps "ces pouvoirs ne pouvaient être illimités que pour la partie politique et militaire, c'est-à-dire pour tout ce qui devait tendre à faire reconnaitre le Gouvernement de Juillet par l'armée et assurer la conquête. Hors de ces points capitaux, il était évident que les ministres d'un roi constitutionnel n'avaient pu déléguer un pouvoir absolu pour l'administration civile $»^{43}$. Le jugement est d'autant plus intéressant que l'Algérie y est qualifiée de « colonie » alors qu'elle n'est pas reconnue comme étant officiellement sous souveraineté française avant 1834. De même, la levée d'une contribution extraordinaire sur la population d'Alger le 7 janvier 1832, bien que jugée illégale par le ministère de la Guerre, est justifié par l'inspecteur général des Finances à Alger par le fait que "Alger n'est pas sous le régime colonial où la législation s'établit par des ordonnances, ni sous la loi française qui n'a pas été publiée. Or dans cette position, les actes émanés de l'autorité militaire ou civile, dans l'étendue de leurs pouvoirs, remplissent toutes les conditions nécessaires de la légalité $\gg{ }^{44}$. Qu'entendre donc par le mot de colonie avant 1834 ? Le terme renvoie dans les écrits de Clauzel et dans le jugement non à une définition juridique, mais au fait de domination et de possible exploitation économique. Seul le traité de capitulation conclut entre le général de Bourmont et le Dey le 5 juillet 1830 est ainsi théoriquement reconnu comme fondement juridique de la relation entre l'autorité militaire et la population, bien qu'il soit dans les faits bafoués par de nombreux actes illégaux ${ }^{45}$. C'est de fait de l'interprétation des agents de l'autorité militaire sur place qu'émerge progressivement une pratique coloniale de facto, résumée ainsi par un sous-intendant de Bône en septembre 1833 : « on a pris l'Afrique pour mettre fin à la piraterie dans un premier but, mais le second est de peupler la côte d'Afrique de Français $»^{46}$.

La question de la transmission des modèles juridiques suppose ainsi de considérer le bagage d'expériences des principaux acteurs de la domination dans les premiers mois de la conquête. Plus que la culture juridique classique, les pratiques des guerres et d'occupations territoriales de la Révolution et du Premier Empire constituent un ensemble expérienciel commun. Le baron Volland, intendant civil à Alger en 1830 a bien été avocat au parlement de Dijon avant la Révolution, mais il a accompli l'essentiel de sa carrière comme intendant militaire et ministère public des tribunaux militaires sous l'Empire ${ }^{47}$. Pierre Genty de Bussy, intendant civil de 1832 à 1834, fut aussi auparavant intendant militaire tandis que LouisPhilibert d'Aubignosc, nommé commissaire de police d'Alger, a certes déjà exercé cette fonction sous l'Empire mais sans formation autre que purement militaire ${ }^{48}$. Louis-André Pichon, intendant civil de décembre 1831 à mai 1832, également ancien administrateur militaire, se démarque par sa carrière consulaire qui lui a permis de connaitre entre autres les États-Unis et Saint-Domingue (Haïti), ce qui l'incite probablement à s'inspirer de la justice consulaire $^{49}$. La concentration des pouvoirs administratifs et judiciaires dans les mains du

\footnotetext{
${ }^{43}$ Estoublon 1890 : année 1832, p. 6, « Pelissier, Clauzel et Volland contre Ministre de la guerre, Conseil d’État, 30 août $1832 »$.

${ }^{44}$ ANOM, 18 MIOM 30 - 1 E 82/4, lettre du 30 janvier 1832, Compte-rendu du conseil de gouvernement du 16 mars 1832.

${ }^{45}$ Dumasy 2015. Sur les abus, cf. ANOM, 18 MIOM 30 - 1 E 82/4, lettre du 30 janvier 1832, qui mentionne des exécutions capitales sans cadre légal à Oran contre l'avis de l'intendant civil.

${ }^{46} 18$ MIOM 25, liasse 66.1., rapport cité.

${ }^{47}$ Sarrut \& Saint-Edme, $1838: 108$

${ }^{48}$ Sur les diverses attributions administratives dans les premiers mois de l'occupation française, cf. Volland 1831.

49 Pichon 1833, rappelle p. 43 qu'il a « rempli des emplois politiques dans trois pays occupés par nos armes, d'abord en Hollande et en Suisse auprès des généraux Joubert, Lecourbe et Morceau, et ensuite en Allemagne au
} 
commandant en chef, qui obtient les pouvoirs de haute police par l'ordonnance du 6 décembre 1831 , donne plus de poids encore à la seule culture militaire ${ }^{50}$. Hors des villes occupées, l'armée est du reste seule compétente, ce qui laisse libre court à des pratiques inspirées des guerres de l'Empire dans laquelle ont combattu tous les généraux en chef qui se succèdent à Alger jusqu'en 1834. Le massacre de la tribu des El Ouffia le 6 avril 1831, sur simple présomption de vol, rappelle les représailles collectives de la guerre d'Espagne de 1808 à $1814^{51}$. Seul Clauzel, qui a combattu à Saint-Domingue en 1802 et qui a une expérience directe des plantations américaines et antillaises, a une connaissance du monde colonial ${ }^{52}$. C'est celle-ci qui l'incite sans doute à voir en l'Algérie une possible colonie agricole soumise à une exception juridique, sur le modèle de la constitution de 1799 envers les colonies et les lois sur l'esclavage de $1802^{53}$. Sans qu'un lien direct ne puisse être démontré dès ce moment, il n'est pas impossible de penser que le maintien d'une justice différenciée et expéditaire s'appuie aussi sur l'expérience des colonies militaires en Illyrie ${ }^{54}$. Y. Urban décèle par ailleurs une filiation avec l'expédition d'Égypte dans l'idée de mission civilisatrice par le droit, alliant séparation juridique dans le présent et proclamation d'une fusion par la loi dans l'avenir ${ }^{55}$. La variabilité des registres du droit est de fait expressément revendiquée comme étant adaptée aux circonstances locales. Si l'idée de «rendre» les habitants de la Régence à «leurs juges naturels » ${ }^{56}$ conduit l'administration coloniale à reprendre en partie la pratique ottomane, les conceptions de ce que doit être le droit en Algérie empruntent donc aussi à un espace de représentations reliant les États-Unis, les Antilles, l'Europe et l'Empire ottoman en fonction des situations.

Cette légitimation de l'exception repose sur quelques postulats que l'on retrouve pendant toute la période coloniale, dans lesquels l'idée de justice est invoquée pour justifier la dérogation au droit métropolitain. L'intendant Volland, en 1831 en offre une des premières formulations : il s'appuie sur les fictions de la demande (la justice d'exception, y compris les pratiques violentes, répondrait au souhait de la population et s'inscrirait dans le respect des pratiques locales); de l'incapacité (les indigènes ne seraient pas en mesure de s'administrer eux-mêmes); de l'absence de véritable justice avant la conquête, et enfin celle, déjà mentionnée, de la fusion juridique dans un avenir indéterminé ${ }^{57}$. Ces représentations cependant

service du royaume de Westphalie » et p. 78 qu'il a accompli « dix années de résidence comme agent politique ou consulaire aux États-Unis, plusieurs missions politiques en Europe, un séjour à Saint-Domingue dans son plus beau temps, une mission aux îles du vent, et une toute récente à Saint-Domingue, où j'ai vu les tristes résultats de nos vingt ans d'erreur ». Voir aussi Esquer, 1912.

${ }^{50}$ Lekéal 2005 : 22.

${ }^{51} \mathrm{Ibid} ., 131-135$. Pichon considère que le tribunal était incompétent en la matière et note par ailleurs que la défense est assurée par un avocat commis d'office ne parlant pas arabe. Sur la répression envers la population civile en Espagne, cf. Lentz 2004 : 190.

${ }^{52}$ Seul Clauzel a combattu à Saint-Domingue en 1802, avant d'être actionnaire de Société de la vigne et de l'olivier en Louisiane, où il côtoie des anciens planteurs venus de Saint-Domingue. Sur ce dernier aspect, Saugera 2011 : 183 et 194 en particulier.

${ }^{53}$ Niort \& Richard 2014.

${ }^{54}$ Brun 2015 : 153 fait le lien entre l'expérience des confins militaires croates et les projets de colonisation militaire de Bugeaud en 1838.

${ }^{55}$ Urban 2014.

${ }^{56}$ L'expression est de Volland $1831: 11$.

${ }^{57}$ Volland $1831: 9$ : « le cours de la justice se trouvait interrompu par la chute du gouvernement turc ; cette population industrielle, accourue de toute part pour se placer sous la juridiction de nos armes, réclamait une juridiction spéciale ». L'idée est reprise par Robineau de Bougon qui écrit que « dès les premiers jours de la conquête les habitants nous demandèrent un joug; ils le demandaient moins dur que celui des Turcs, mais ils sentaient qu'il leur en fallait un pour réprimer le brigandage commun chez les peuples belliqueux » (Robineau de Bongon, 1835 : 12). Le rapport du sous-intendant civil de Bône du 21 septembre 1833 montre une autre variante de ces postulats, selon laquelle « les bédouins sont menteurs et voleurs, les maures sont corrupteurs. La fraude est leur industrie de prédilection». ANOM, 18 MIOM 25, Liasse 66-1. Sur la fiction de la fusion juridique idéale et le régime de l'exception, cf. Volland, ANOM, 18 MIOM 25, rapport du 8 novembre 1833, qui note qu'il n'est « pas moins difficile, d'instituer petit à petit, et insensiblement, un peuple grossier dans nos idées d'ordre et de 
ne sont pas propres à l'univers colonial, comme le montrent les comparaisons faites entre les habitants d'Algérie et les Bretons ou Normands jusque dans les années 1840, perçus comme rétifs à la modernité administrative et juridique ${ }^{58}$. Ils ne forment pas non plus les bases d'un discours cohérent, ces postulats servant aussi bien à plaider en faveur d'un rapprochement que de l'extermination des populations locales selon l'orientation des acteurs ou celle de l'auditoire. Mais ils ont l'utilité de réinscrire des mesures hétérogènes dans un idéal proclamé de justice, de rectitude et d'humanité qu'il faut interroger comme partie intégrante du processus colonial. L'insistance sur ces notions, qui innervent les rapports des administrateurs, vise à donner une cohérence à ce qui n'en a pas. Ce qui interroge n'est pas la violation du droit et de la justice en soi, mais l'invocation récurrente de ces notions comme valeurs de référence.

\section{Les limites de l'espace du droit}

Quelle place a dès lors le droit dans le processus colonial? La justice en Algérie, notamment envers les musulmans et les juifs, repose sur un droit réduit si on le compare aux évolutions dans la métropole ${ }^{59}$. Exubérant dans son volet répressif, ce droit est à la fois peu défini dans ses objets et limité dans ses institutions ${ }^{60}$. Rappelant cependant la complexité des procédures juridiques et la lourdeur du contrôle parlementaire, L. Blévis note que « l'argument d'une légitimation artificielle n'épuise pas le mystère de la nécessité de passer par le droit $»^{61}$. Sortir de l'impasse de la dichotomie entre intentions proclamées et arbitraire constaté suppose dès lors de replacer le droit dans une analyse plus générale de la gouvernementalité coloniale.

Le recours à la procédure judiciaire n'apparait que comme une des options de la résolution de conflits et d'imposition d'un ordre colonial. La praticité et l'urgence sont ainsi abondamment évoqués pour justifier les violences et les séquestres au nom du droit de guerre, d'autant que les territoires d'Algérie sont sous l'autorité directe du ministère de la Guerre jusqu'en $1848^{62}$. Le cas de Collombon, juge français à Alger accusé en 1832 d'avoir soustrait une femme mariée - et sa sœur - à son mari, illustre un autre volet de la logique de limitation des procédures légales au nom d'une raison pratique politique. Contre la possibilité d'une action en justice qui porterait atteinte au prestige de l'autorité et risquerait d'entrainer des troubles, le général en chef Savary duc de Rovigo décide seul de soumettre la sœur à la juridiction du qadi et de renvoyer la femme à son mari, sans jugement. L'affaire donne lieu à un échange entre l'intendant civil Pichon et le général, puis entre Pichon et le ministre de la Guerre (qui est alors aussi le président du Conseil). Pour Pichon, qui plaide pour un jugement régulier, "nous sommes de droit et devons être de fait les conservateurs des lois qui les garantissent à chacun d'eux $»^{63}$. Pour le ministre au contraire, ce cas d'adultère serait, "dans ces circonstances », moins « une affaire civile et judiciaire qu'une affaire de gouvernement. S'il y a des irrégularités elles sont suffisamment couvertes par la nature du délit », car « il est bien difficile de conserver

justice. Pour cela, il faut que l'autorité administrative réside tout entière dans un chef militaire, car il n'y a qu'une administration armée qui puisse opérer ou du moins préparer cette métamorphose, puisqu'elle doit tour à tour user des moyens de force et de persuasion ». Il est évidemment impossible de relever toutes les occurrences de ce genre de propos.

${ }^{58}$ Dumasy 2017.

${ }^{59}$ Ewald 1986. Sur la simplification de la justice en Algérie, cf. Durand 2005 et 2015.

${ }^{60} \mathrm{La}$ séparation des juridictions conduit à considérer le droit civil musulman ou israélite comme ne relevant pas de la compétence première de l'autorité coloniale, même si celle-ci se réserve rapidement un droit d'intervention. De même, les possibilités d'appel, voire de défense, sont réduites et les procédures simplifiées au pénal, ce qui aboutit à l'affirmation de la figure du juge unique. Cf. Durand 2005 et Lekéal 2005. Inversement, les infractions prévues par le « code de l'indigénat » sont multiples (Merle 2004).

${ }^{61}$ Blévis 2013 : 93.

${ }^{62}$ Lekéal $2005: 27$; Frémeaux 2005.

${ }^{63}$ ANOM, 18 MIOM 30, 1 E 82/4, lettre de Pichon au général Savary, 30 janvier 1832. 
l'ordre et la puissance dans une colonie en adoptant rigoureusement la législation de la métropole ». Ainsi " nous pouvons choisir entre les deux directions à donner à cette affaire, ou l'action gouvernementale, ou celle d'une accusation juridique. Nous avons préféré la première, si la seconde eut été plus opportune ». C'est cette même logique qui conduit le ministre à conseiller « un régime exceptionnel dans ce pays » tempéré de « justice et de modération ${ }^{64}$.

L'invocation de la justice renvoie ici à une capacité d'adaptation par définition imprécise, où peuvent alterner aussi bien la tempérance que la violence. Cette « polysémie du principe de légalité ${ }^{65} \gg$ favorise de fait d'autres acteurs que ceux du droit stricto sensu, dans un contexte marqué non seulement par la pérennisation de l'état de guerre, mais aussi par des conflits de légitimation entre les agents de l'autorité. Le général Savary peut ainsi prétendre agir au nom de la «justice et de l'humanité » en confisquant en février 1832 des terres près d'Alger pour les redistribuer à des colons miséreux, contre l'avis de l'intendant civil Pichon qui plaide au contraire pour le respect de la propriété et des procédures légales d'expropriation comme une « obligation de justice universelle » antérieure à toute forme de constitution ${ }^{66}$.

La mobilisation du répertoire sémantique du droit est d'autant plus importante que la remise en cause de l'administration militaire génère des stratégies de justification à destination du public français ${ }^{67}$. Pour autant, la justice en tant que telle n'occupe qu'une place restreinte dans les rapports internes de l'administration et dans les multiples publications du temps sur l'Algérie si on la rapporte aux considérations sécuritaires et économiques. Le commerce et la mise sur le marché des terres sont ainsi fréquemment présentés comme des ressorts de pacification d'autant plus efficaces qu'ils créeraient les conditions d'une communauté d'intérêts. Pour le sous-intendant civil à Bône en 1833, qui considère que « la première de toutes les difficultés [...] est de faciliter aux colons le moyen de se procurer des bras à bon marché », la question de la sécurité est subsumée par celle de l'intérêt, selon l'idée que « une légère augmentation de profit attirera les tribus les plus éloignés ». La politique de la France doit donc rester fondée « sur ce peu de mots, laissez faire, laissez passer ${ }^{68}$. Un sous-intendant militaire propose pour sa part la même année de " céder Alger à une grande compagnie européenne » qui « serait souveraine » et à qui « appartiendrait le droit de proclamer le code judiciaire ${ }^{69}$. Ces positions sont paradoxales en ce qu'elles occultent les conséquences sociales de l'accaparement de terres qui alimentent le rejet de l'occupation française. Si elles ne reflètent pas nécessairement la position du gouvernement, elles illustrent l'articulation entre le droit et l'économie opérée sur le terrain par certains responsables, d'autant que c'est en grande partie sur les aspects économiques que se cristallisent les débats au parlement sur l'opportunité de rester en Algérie.

Prendre en considération non seulement les textes juridiques et l'espace des tribunaux, mais aussi la façon dont les acteurs s'emparent des idées de droit ou de justice pour développer des modes de légitimation et des principes d'action hors du domaine juridique proprement dit, permet ainsi par effet retour de repenser la place du droit comme institution dans l'économique générale des modes de domination coloniales. L'éclatement du droit en législations différenciées dès 1830 et les justifications de l'exception, y compris par l'arbitraire

\footnotetext{
${ }^{64}$ Ibid., 1 E 82/3, lettre à l'intendant Pichon, 16 février 1832.

${ }^{65}$ Lekéal 2005 : 28.

${ }^{66}$ Dumasy 2016 : 48-50.

${ }^{67}$ Les généraux Clauzel, Berthezène, Rovigo ont rapidement cherché à légitimer leur action par des publications. C'est le cas aussi des intendants Pichon, Volland et Genty de Bussy ou du directeur des finances Blondel. Les « commissions d'Afrique » de 1833 et 1843 , de même que les discussions annuelles à la Chambre sur le budget alloué à l'Algérie constituent des moments importants de ces prises de parole.

${ }^{68}$ ANOM, 18 MIOM 25, Liasse 66-1, rapport du 21 septembre 1833, op. cit. Souligné dans le texte.

${ }^{69}$ Flandin $1833: 11-15$.
} 
le plus violent, n'empêchent pas l'appel à des principes supérieurs puisant à des registres différents tels que la civilisation, le marché ou le respect supposé de la volonté des habitants face à l'indétermination des règles politiques. Les expériences personnelles des agents locaux de l'autorité française imposent de suivre non seulement l'application des textes, mais aussi la diversité des univers de référence d'acteurs dont la culture juridique peut être d'autant plus limitée que la connaissance des modes de justice locaux est par ailleurs très faible. L'étude de la circulation des modèles et des modes d'énonciation, encore peu explorées par la recherche, permettrait de mieux comprendre la façon dont se diffuse un habitus juridique dans le personnel militaire dont il est aussi nécessaire de repérer les limites.

Suivre le rythme des réformes du droit ne peut donner dès lors qu'un aperçu restreint des enjeux y afférant. Les conflits de compétence, la faiblesse des moyens, le désir de promotion comme les convictions des divers représentants du pouvoir génèrent l'édiction de registres concurrentiels de normes qui restent encore à étudier, non seulement pour elles-mêmes, mais pour appréhender plus finement la façon dont se noue le rapport colonial avec les populations. L'approche par le droit ne s'oppose pas à une histoire « par le bas » du fait colonial, elle peut la compléter. De même, les débats parlementaires ont des effets de contrainte qui orientent la façon de penser le droit. En portant la question de la présence française en Algérie sur le terrain des coûts et des bénéfices économiques et, dans une moindre mesure pour cette période, stratégiques, ils conduisent à valoriser notamment les aspects budgétaires. Or les discours sur les avantages commerciaux qui émergent dans la période 1830-1834 laissent place dans la seconde moitié des années 1830 à la focalisation sur la libéralisation des propriétés face aux résistances produites par le droit local, qui en limitent le marché. Lors des débats parlementaires sur l'Algérie en 1836 et 1838, le directeur des Finances à Alger, Léon Blondel, peut ainsi décrire les possessions d'Afrique comme un territoire « où la force donne seule des droits » ${ }^{70}$, et où il est nécessaire de s'affranchir de « la justice telle que nous l'entendons avec ses formes lentes, ses arguties de barreau, ses sévérités préventives ${ }^{71}$. Si l'arrêté du $1^{\text {er }}$ décembre 1840 qui confirme les séquestres et étend le droit de confiscation lui apparaît comme une "législation monstrueuse » dans laquelle « le Gouvernement prend tout, est juge et partie dans cette cause », il n'en est pas moins une " nécessité » imposée par le droit de propriété local et la guerre. Aussi « il n’y avait qu'un moyen à employer pour pouvoir rester équitable, c'était l'arbitraire ${ }^{72}$.

\section{Bibliographie :}

BAYART Jean-François, Les Études postcoloniales, un carnaval académique, Paris, Karthala, 2010.

BERTRAND Romain, «Politiques du moment colonial. Historicités indigènes et rapports vernaculaires au politique en 'situation coloniale' ", Questions de recherche, 26, 2008, p. 1-49.

BERTRAND Romain, « Histoire d'empires. La question des continuités du colonial au prisme de l'histoire impériale comparée ", in P. R. Baduel, Chantiers et défis de la recherche sur le Maghreb contemporain, Karthala, IRMC, 2009, p. 537-562.

\footnotetext{
${ }^{70}$ Blondel $1836: 35-51$.

${ }^{71}$ Blondel $1838: 112$ et 117.

72 ANOM F80/1084, Rapport au conseil, 8 août 1842. Ce passage est développé plus amplement dans Dumasy 2016.
} 
BERTRAND Romain, « Norbert Elias et la question des violences impériales. Jalons pour une histoire de la «mauvaise conscience » coloniale », Vingtième Siècle, 2010, 2-106, p. 127-140.

Blais Hélène, Fredj Claire, Thénault Sylvie, « Désenclaver l'histoire de l'Algérie à la période coloniale », Revue d'Histoire Moderne et Contemporaine, 2018, 63-2, p. 7-13.

BLÉVIS Laure, « La citoyenneté française au miroir de la colonisation. Étude des demandes de naturalisation des « sujets français » en Algérie coloniale », Genèses, 2003/4 (nº53), p. 25-47.

BLÉVIS Laure, «La situation coloniale entre guerre et paix. Enjeux et conséquences d'une controverse de qualification », Politix, 2013, 4-104, p. 87-104.

BLONDEL Léon, Aperçu sur la situation politique, commerciale et industrielle des possessions françaises dans le Nord de l'Afrique au commencement de 1836, Paris, Imprimerie royale, 1836.

BlONDEL Léon, Nouvel aperçu sur l'Algérie. Trois nécessités en Afrique : conserver, pacifier, coloniser, Paris, Delaunay, 1838

BonTEMS Claude, Le droit musulman algérien à l'époque coloniale. De l'invention à la codification, Slatkine, 2014.

BonTEMs Claude, L'Algérie, ses institutions, son droit à l'épreuve de la colonisation, Alger, Bouchene, 2018

BRAS Jean-Philippe (dir.), Faire l'histoire du droit colonial. Cinquante ans après l'indépendance de l'Algérie, IISMM-Karthala, 2015.

BRUN Jean-François, "Confins militaires et régiments croates de l'armée napoléonienne », in Jacques-Olivier Boudon, Les provinces illyriennes dans l'Europe napoléonienne (1809-1813), Paris, 2015, SPM, p. 137-154.

BRunet-La Ruche Bénédicte \& MANièRe Laurent, «De l'« exception » et du "droit commun » en situation coloniale : l'impossible transition du code de l'indigénat vers la justice indigène en AOF », in B. Piret, C. Braillon, L. Montel, P.-L. Plasman (dir.), Droit et Justice en Afrique coloniale: Traditions, productions et réformes. Bruxelles, Presses de l'Université Saint-Louis, 2014, p. 117-141.

CHERCHARI Mohamed Sahia, «Indigènes et citoyens ou l'impossible universalisation du suffrage », Revue française de droit constitutionnel, 2004, 60, p. 741-770.

Collot Claude, Les institutions de l'Algérie durant la période coloniale (1830-1962), Paris, C.N.R.S. et Alger, Office des publications universitaires, 1987.

DUMASY François, «Propriété foncière, libéralisme économique et gouvernement colonial : Alger, 1830-1840 », Revue d'histoire moderne \& contemporaine, 2016/2, n 63-2, p. 40-61.

DUMASY, François, «Propriété et société coloniale. La Commission de colonisation et la Mitidja en 1842-1843 », in D. Guignard, Propriété et société en Algérie contemporaine. Quelles approches ?, Aix-en-Provence, IREMAM, 2017, http://books.openedition.org/iremam/3637. 
DURAND Bernard, "Originalité et exemplarité de la justice en Algérie (de la conquête à la seconde guerre mondiale) », Histoire de la justice, 2005/1, n 16, p.45-74.

DURAND Bernard, Introduction historique au droit colonial, Economica, 2015

ESQUER Gabriel, « Les débuts de l'administration civile à Alger. Le personnel », extrait de $L a$ Revue africaine, Alger, A. Jourdan, 1912.

Estoublon Robert, Bulletin judiciaire de l'Algérie. Jurisprudence algérienne de 1830 à 1876, tome premier, 1830-1848, Alger, A. Jourdan, 1890.

EWALD François, L'État providence, Grasset, 1986.

Flandin J.-B, Régence d'Alger. Peut-on la coloniser ? Comment ?, Paris, Féret, 1833.

FRÉMEAUX Jacques, « Justice civile, justice pénale et pouvoirs répressifs en territoire militaire (1830-1870), Histoire de la justice, 2005/1, n¹6, p. 31-44.

GÉRARD-LOISEAU Sandra, RENUCCI Florence, Les discours sur le droit et la justice au Maghreb pendant la période coloniale, XIXe-XXe s.), Centre d'histoire judiciaire, 2011

GoBE Éric, Les avocats en Tunisie de la colonisation à la révolution (1883-2011), Paris, Karthala, 2013.

GRANGAUD Isabelle \& OUALDI M'hamed, « Tout est-il colonial dans le Maghreb ? Ce que les travaux des historiens modernistes peuvent apporter", Revue d'histoire moderne \& contemporaine, 2016, 63-2, p. 133-156.

KenNEDy Dunkan, "Three Globalizations of Law and Legal Thought: 1850-2000 », in D. Trubek \& A. Santos, The New Law and Economic Development: A Critical Appraisal, Cambridge, Cambridge University Press, p. 19-73.

Le Cour GRANDMAISON Olivier, Coloniser, exterminer : sur la guerre et l'État colonial, Paris, Fayard, 2005

Le Cour Grandmaison Olivier, « Du droit colonial », Droits 2006/1, n 43, p. 123-140.

LENTZ Thierry, Nouvelle histoire du premier Empire, vol. II. L'effondrement du système napoléonien, 1810-1814, Fayard, 2004.

MANN, Gregory, "What was the "indigénat"? The "Empire of Law" in French West Africa" ", The Journal of African History, 2009, 50-3, p. 331-353.

MERLE Isabelle, «De la « légalisation» de la violence en contexte colonial. Le régime de l'indigénat en question », Politix, 2004, 66, p. 137-162.

Merle Isabelle \& Muckle Adrian, L'indigénat. Genèses dans l'empire français. Pratiques en Nouvelle Calédonie, Paris, CNRS édition, 2019. 
NIORT Jean-François et RICHARD Jérémy, « De la Constitution de l'an VIII au rétablissement de l'esclavage (1802) et à l'application du Code civil dans les colonies françaises (1805) : le retour d'un droit colonial réactionnaire sous le régime napoléonien », in F. Régent ; J.-F. Niort, P. Serna, Les colonies, la Révolution française, la loi, Rennes, PUR, 2014, p. 165-177.

PICHON Louis-André, Alger sous la domination française, Paris, Barrois et Duprat, 1833.

PITTS Jennifer, Naissance de la bonne conscience coloniale. Les libéraux français et britanniques et la question impériale (1770-1870), éd. de l'atelier, 2008.

PouchePADASS Jacques, «Jean-François Bayart. Les Études postcoloniales. Un carnaval académique », Afrique contemporaine, 2010/3 (n²35), p. 149-152.

RENUCCI, Florence, « Citoyenneté et statut personnel dans l'Algérie coloniale (1865-1944)», in M. Augeron et P. Even, Les étrangers dans les villes-ports atlantiques. Expériences françaises et allemandes (XVe-XIXe siècles), Paris, Les Indes Savantes, 2010, p. 95-108.

RENUCCI Florence, « Les chantiers de l'histoire du droit colonial. Introduction»,Clio@Themis, $\mathrm{n}^{\circ} 4,2011$, p. 1-7.

RENUCCI Florence, «Les magistrats dans les colonies : un autre apprentissage des normes juridiques ? », Cahiers de la Justice, 2016/4, p. 493-501.

Robineau de Bougon, Chambre des députés, séance du 26 mai 1835. Opinion de M. Robineau de Bougon, député de la Loire inférieure, sur la question d'Alger, Paris, Imp. Le Normant, sd.

RousSEAUX, Xavier. "Introduction. Vers une histoire post-postcoloniale de la justice et du droit en situation coloniale ?», in B. Piret et allii, Droit et Justice en Afrique coloniale: Traditions, productions et réformes, Bruxelles, Presses de l'Université Saint-Louis, 2014, p. 926.

SAADA Emmanuelle, " The empire of law: dignity, prestige, and domination in the "situation" », French Politics, Culture and Society, 2002, 20, p. 98-120.

SAADA Emmanuelle, « La loi, le droit et l'indigène », Droits, 2006/1, nº43, p. 165-190.

SAADA Emmanuelle, « Penser le fait colonial à travers le droit en 1900 », Mil neuf cent, 2009, 1-27, p. 103-116.

SARrut G. \& SAINT-EDME B., Biographie des hommes du jour, t. IV, $1^{\text {re }}$ partie, Paris, Pilout, 1838 .

SAUGERA Éric, Reborn in America. French exiles and refugees in the United States and the Vine and Olive Adventure, 1815-1865, Tuscalosa, University of Alabama Press, 2011.

SESSIONS Jennifer, By Sword and Plow. France and the Conquest of Algeria, Ithaca (N.Y.), Cornell University Press, 2011.

SURKIS Judith, «Propriété, polygamie et statut personnel en Algérie coloniale, 1830-1873 », Revue d'histoire du XIXe siècle, 2010, 41 (http://journals.openedition.org/rh19/4041). 
SURKIS Judith, «An Effective and Affective History of Colonial Law », in J. Desautels-Stein \& C. Tomlins, Searching for Contemporary Legal Thought, Cambridge, Cambridge University Press, 2017, p. 238-255.

SuRKIS Judith, Sex, Law and Sovereignty in French Algeria, 1830-1930, Cornell University Press, 2019.

ThÉNAult Sylvie, Une drôle de justice. Les Magistrats dans la Guerre d'Algérie, Paris, La Découverte, 2001.

THÉNAUlT Sylvie, Violence ordinaire dans l'Algérie coloniale. Camps, internements, assignations à résidence, Paris, O. Jacob, 2011.

URBAN Yerri, « L'ordre du jour du 10 vendémaire an IX [2 octobre 1800] relatif à l'organisation de la justice en Égypte : vers un nouveau droit colonial », in F. Régent et alii, Les colonies, la Révolution française, la loi, Rennes, PUR, 2014, p. 149-164.

VATIN Jean-Claude, "Retour sur les spécificités algériennes », in Ph. Bras, Faire l'histoire du droit colonial, cinquante ans après l'indépendance de l'Algérie, IISMM-Karthala, 2015.

MeYNIER Gilbert, VIDAL-NAQUet Pierre, "Coloniser Exterminer : de vérités bonnes à dire à l'art de la simplification idéologique », Esprit, décembre 2005, p. 162-176.

Volland Paul Ambroise, Notice sur le mode de gouvernement provisoirement établi dans le royaume d'Alger, $8^{\circ}$, janvier 1831 . 